\title{
Effect of Disease Modifying Agent on Oxidant and Antioxidant Status in
}

\section{Rheumatoid Arthritis}

\author{
Kowsalya Ramprasad ${ }^{1}$, Ashok Prabhu ${ }^{2}$
}

\author{
Affiliations: \\ ${ }^{1}$ Department of Biochemistry Institute of \\ NephroUrology \\ Victoria hospital campus. Bangalore. \\ ${ }^{2}$ Department of Biochemistry Kasturba Medical \\ College, Centre for Basic Sciences, Bejai. \\ Mangalore.
}

\author{
Correspondence to: \\ Kowsalya Ramprasad \\ Department of Biochemistry Institute of \\ NephroUrology \\ Victoria hospital campus. Bangalore. \\ Email: r.kowsalya@gmail.com
}

\section{How to cite this Article:}

Effect of Disease Modifying Agent on Oxidant and Antioxidant Status in Rheumatoid Arthritis. Kowsalya R. Prabhu A. Ann. Clin. Chem. Lab. Med. 2017:3(2); 11-15

DOI: http://dx.doi.org/10.3126/acclm.v3i2.20738

\section{(C) 2017 Nepalese Association for Clinical Chemistry}

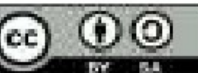

This work is licensed under a Creative Commons Attribution-Share Alike 4.0 International License.

\begin{abstract}
BACKGROUND

Rheumatoid Arthritis is a chronic disease and a leading cause of disability in people with advancing age. Disease modifying antirheumatic drugs have become the mainstay of management in rheumatoid arthritis. Among these drugs, methotrexate the most frequently used agent has a proven anti-inflammatory action and is known to slow down the progression of the disease. Hence the present study is undertaken to evaluate the effect of methotrexate on the oxidant and antioxidant status in patients with rheumatoid arthritis on methotrexate.

\section{METHODS}

A total of 40 rheumatoid arthritis patients and 40 controls were enrolled for the study. The rheumatoid arthritis patients were further divided into patients on methotrexate (group I) and patients without methotrexate (group II). The lipid peroxidation index -Malondialdehyde and total antioxidant activity, superoxide dismutase and glutathione levels were analyzed in all the participants.
\end{abstract}

\section{RESULTS}

A significant increase in total antioxidant activity $(\mathrm{p}=0.048)$ and decrease in lipid peroxidation ( $\mathrm{p}=0.04)$ was found in methotrexate treated arthritis patients compared to patients without methotrexate.A significant negative correlation ( $p=0.01$ ) was found between MDA and total antioxidant activity in both groups of patients whereas, the antioxidant enzyme superoxide dismutase was positively correlated with lipid peroxide levels.

\section{CONCLUSION}

In conclusion low dose methotrexate seems to act as an effective anti-inflammatory drug and may also play an important role in minimizing the oxidative stress in rheumatoid arthritis. Serum total antioxidant activity is significantly raised in subjects with altered liver function profile.

\section{KEY WORDS:}

Total antioxidant activity, Liver Function test, Transaminase 


\section{NTRODUCTION}

Rheumatoid Arthritis (RA) still remains one of the most puzzling and poorly treated diseases of humans. It is a heterogeneous disorder with a spectrum of clinical severity ranging from mild arthritis to crippling destruction of joints and life threatening involvement of internal organs. It's a universal disease with a prevalence of $0.75 \%$ in adult Indian population ${ }^{1}$.The long-term prognosis is poor, $80 \%$ of affected patients are disabled by 20 years and life expectancy is reduced by an average of 3 to 18 years. Patients with extra articular manifestations have increased mortality rate as well as more severe disability $^{2}$.For many individuals RA is a long lasting debilitating disease.

The focus of RA is the synovial lining, a highly vascularised tissue which surrounds the bones of the joint. Many reports suggest that lipid peroxidation product, malondialdehyde (MDA) a 3-carbon dialdehyde, which can form cross linkages in proteins is increased both in plasma and synovial fluid of patients with RA. Though RA has been categorized as a connective tissue disease and not recognized as a disease of oxidative stress, growing evidence suggests that production of free radicals within the inflamed joint can produce autoimmune phenomena and connective tissue destruction 3,4

Traditionally treatment of RA starts with non -steroidal anti-inflammatory drugs and/or corticosteroids. Then in later stage as the disease progresses, disease-modifying antirheumatic drugs (DMARDs) are added to the patient treatment regime. But recently, studies have shown that DMARDs alter the disease course playing a significant role in slowing the joint destruction. So treatment modalities have changed with patients been started early on with DMARDs. A number of DMARDs exist, of which low dose methotrexate is the most frequent and first choice. Other commonly used drugs are sulfasalazine, leflunomide, gold salts, antimalarial chloroquinine and azathioprine. Some of these drugs have been shown to possess an inhibitory effect on reactive oxygen species production ${ }^{5,6}$. So the aim of the present study is to elucidate the possible effect of
DMARD - methotrexate on lipid peroxidation and antioxidant status in patients suffering from RA.

\section{METHODS}

The study group consisted of 40 patients between the ages 27 to 69 years of both sexes (26 females and 14 males). These patients were selected from rheumatology clinic of Kasturba Medical College hospital and other hospitals in Mangalore. All the patients fulfilled the ACR revised criteria used for diagnosis of $\mathrm{RA}^{7}$. The median disease duration was 5.4 years. Normal age and sex matched individuals were taken as controls. All subjects were informed about the objectives of the study and informed consent was obtained. Patients with acute exacerbation of the illness and those on corticosteroids were not included in the study but were on NSAID for pain relief. The study was approved by the institutional ethics committee.

The Study group of RA patients were grouped as follows:

Group I - consisted of 20 RA patients treated with Methotrexate.

Group II - consisted of 20 RA patients not treated with Methotrexate / DMARDs.

$5 \mathrm{ml}$ venous blood was collected in vacutainers under aseptic precautions from both patients and controls. Plasma MDA was measured using the modified method of Satoh ${ }^{8}$ and total antioxidant activity (TAA) by the method of Koracevic et al ${ }^{9}$. Superoxide Dismutase (SOD) was estimated by the method of Beauchamp and Fridovich ${ }^{10}$ and whole blood glutathione (GSH) was determined by the method of Beutler et al ${ }^{11}$. Data were statistical analyzed by Unpaired t-test and Pearson's Correlation and expressed in terms of $p$ value.

\section{RESULTS}

The marker of lipid peroxidation - MDA $(p=0.0001)$ was significantly increased in RA patients compared to controls. Whole blood GSH ( $\mathrm{p}=0.005) \&$ total antioxidant activity levels $(\mathrm{p}=0.0001)$ were decreased significantly in RA patients compared to controls. Superoxide dismutase activity ( $p$ $=0.005$ ) was notably increased in RA patients compared to controls. 


\begin{tabular}{|l|l|l|}
\hline \multicolumn{3}{|c|}{ Table I : Comparison of various parameters between control subjects and RA patients } \\
\hline \multicolumn{1}{|c|}{ Parameters } & Controls $(\mathrm{n}=40)$ & RA patients $(\mathrm{n}=40)$ \\
\hline MDA (nmol/dl) & $154.7 \pm 34.5$ & $196.8 \pm 46.1^{*}$ \\
\hline TAA (mmol/L) & $1.81 \pm 0.32$ & $1.55 \pm 0.17^{*}$ \\
\hline SOD (U/gHb\%) & $4937.38 \pm 961.5$ & $5443.48 \pm 864.54^{* *}$ \\
\hline GSH (mg/dl) & $48.5 \pm 6.64$ & $44.49 \pm 5.1 * *$ \\
\hline & & \\
\hline
\end{tabular}

$* \mathbf{P}<0.0001 ; * * \mathbf{P}<0.001$

There was a significant decrease in lipid peroxidation $(\mathrm{p}=0.04)$ in methotrexate treated RA patients (group I) compared to non-methotrexate treated RA patients (group II). Similarly the total antioxidant activity ( $\mathrm{p}=0.048$ )was also significantly increased in group I patients compared to group II patients. Whole blood glutathione and SOD levels were almost similar but numerically different in both the groups of patients (Table II).

\begin{tabular}{|l|l|l|}
\hline \multicolumn{3}{|c|}{ Table II : Comparison of various parameters among both groups of RA patients } \\
\hline Parameters & Controls(n=20) & RA patients $(\mathrm{n}=20)$ \\
\hline MDA (nmol/dl) & $181.35 \pm 43.5$ & $212.25 \pm 48.7^{*}$ \\
\hline TAA (mmol/L) & $1.62 \pm 0.13$ & $1.49 \pm 0.18^{*}$ \\
\hline SOD (U/gHb\%) & $5268.18 \pm 881.9$ & $5618.78 \pm 909.5$ \\
\hline GSH (mg/dl) & $45.44 \pm 4.22$ & $43.55 \pm 5.8$ \\
\hline & & \\
\hline
\end{tabular}

$* \mathrm{P}<0.05$

A significant strong negative correlation was noted between the total antioxidant activity $(\mathrm{p}=0.01)$ and MDA level whereas a positive correlation between the antioxidant enzyme SOD and MDA was noted in both groups of rheumatoid arthritis patients. The antioxidant GSH was also

\begin{tabular}{|l|l|l|l|l|}
\hline \multicolumn{5}{|c|}{ Table-3 showing the correlation between various parameters in RA patients } \\
\hline Parameters & & TAA & SOD & GSH \\
\hline MDA (nmol/dl) & r & -0.75 & 0.16 & -0.32 \\
\hline & p & $0.01^{*}$ & 0.64 & 0.36 \\
\hline & & & & \\
\hline
\end{tabular}

$\mathbf{r}=$ spearman's correlation 


\section{DISCUSSION}

The excessive production of reactive oxygen species (ROS) can damage cellular components leading to cellular apoptosis. In RA, studies have shown that these ROS can depolymerise hyaluronic acid and oxidize $\operatorname{IgG}$ inducing rheumatoid factor production ${ }^{12,13}$.These ROS also serve as important intracellular signaling molecules that amplify the synovial inflammation-proliferative response. Thus, the enhanced oxidative stress and decreased antioxidant status observed in RA patients indicate the potential for oxidative injury to joints and synovial membrane.

Low dose methotrexate has been proved as an effective drug in controlling the inflammatory manifestations of RA. This is attributed to the targeting of ROS producing centers of the stimulated inflammatory cells leading to their apoptosis. Thus inhibiting their interaction with the inflammatory joint synovium. Methotrexate also seems to reduce the production of pro-inflammatory cytokines and mediators of inflammation especially interleukin-6 and tumor necrosis factor $-\propto^{14}$. The present study demonstrated that in RA patients' use of DMARDs is associated with decrease in inflammation and changes in lipid peroxidation levels.

The total antioxidant activity is a dynamic equilibrium, the cooperation of the antioxidants in the human serum. TAA is indicative of disturbance in the antioxidant system, a result of diminished individual anti-oxidants. Gutteridge JM has shown that patients with RA have altered protein pattern in their serum and synovial fluid, which influences the TAA of these fluids ${ }^{15}$. The observed changes in TAA in methotrexate treated RA patients may be due to better control of inflammation.

Glutathione, a tri-peptide thiol is a natural reservoir of reductive capacity of the cell. It is one of the major endogenous antioxidant. It maintains the thiols of many proteins and reduced forms of other antioxidants such as ascorbic acid $\& \propto$-tocopherol ${ }^{16}$. Superoxide dismutase (SOD) is another free radical scavenging enzyme enhances the otherwise slow spontaneous breakdown of superoxide, forming less toxic hydrogen peroxide which can then interact with GSH and ultimately lead to $\mathrm{H}_{2} \mathrm{O}$ and $\mathrm{O}_{2}{ }^{17}$. Mn-SOD has been found inducible under condition of stress/ inflammation, through the activation of down stream signaling pathways ${ }^{18}$. Manish et al have reported a significant increase in SOD levels in RA patients on NSAIDs ${ }^{19}$.

In our study, RA patients of both group I and II showed significant increase in SOD and significant decrease in GSH in comparison with the controls. A non-significant and numerically different SOD and GSH levels were found among both the groups of RA patients. These non-significant changes in different parameters analyzed among group I and II patients in our study may be due to the small number of cases and difference in severity of illness in the patients.

It has been postulated that dietary intervention with vitamins may reduce oxidative stress and thereby reduce the disease related morbidity and mortality associated with the disorder. Antioxidant defenses limit the damages caused by oxidants, such as those formed during inflammation. In addition, in vitro studies and animal studies have shown that antioxidants also possess anti-inflammatory properties. This implies that anti-oxidative defense mechanisms are of particular importance for patients with RA

In conclusion low dose methotrexate seems to exert anti-inflammatory effects by acting at different levels of pathophysiological cascade. Though the exact mechanism of methotrexate action in RA still remains unclear, it definitely is an effective anti inflammatory drug that helps to contain the oxidative stress mediated injury in rheumatoid arthritis.

\section{ACKNOWLEDGEMENTS}

The authors acknowledge Dr. Prabha Adhikari, Professor,Dept of Medicine. Kasturba Medical College, Mangalore and for her kind support and subjects for their cooperation.

\section{CONFLICT OF INTEREST}

None declared 


\section{REFERENCES}

1. Malaviya AN, Kapoor SK, Singh RR, Kumar A and Pande I.Prevalence of rheumatoid arthritis in the adult Indian population. Rheumatol Int 1993;13:131-134.

2. Scott PL, Symmons DP, Coulton BL and Popert AJ. Long term outcome of treating rheumatoid arthritis after 20 years. Lancet 1987; 1: 1108- 1111.

3. Chaturvedi V, Handa R, Rao DN and Wali JP. Estimation and significance of serum and synovail fluid malondialdehyde levels in rheumatoid arthritis. Ind $\mathbf{J}$ Med Res 1999; 109: 170-174.

4. Droge W. Free radicals in physiological control of cell functions. Physiol Rev 2002;82: 47-95.

5. Acleves-Avila FJ, Medina F and Fraga A. The antiquity of rheumatoid arthritis: a reapprisal. J Rheumatol 2001; 28: 751-757.

6. Kremer JM. Methotrexate and emerging therapies. Rheum Dis Clin North Am 1998; 24: 651-658.

7. Arnett FC, Edworthy SM, Bloch DA etal. The American rheumatism association 1987 revised criteria for the classification of rheumatoid arthritis.Arthritis Rheum 1998; 31: 315-324.

8. Satoh K. Serum lipid peroxide in cerebrovascular disorders determined by a new colorimetric method. Clin Chim Acta 1978;90:37-43.

9. Koracevic D, Koracevic G, Djordjevic V, Andrejevic $\mathrm{S}$ and Cosic V. Method for measurement of antioxidant activity in human fluids. J Clin Pathol 2001;54:356-361.

10. Beauchamp C and Fridovich IM. Superoxide dismutase: improved assays and an assay applicable to acrylamide gels. Anal Biochem 1971;44:276-287.

11. Beutler E, Duron O, Kelly BM. Improved method for the determination of blood glutatione. J Lab Clin Med 1963;61:882-888.

12. Mapp PI, Grooteveld MC and Blake DR. Hypoxia, oxidative stress and rheumatoid arthritis. Br Med Bull 1995;51:419-436.

13. Laurindo IMM, Mello SBV, Cossermelli W. Influence of low doses of methotrexate on superoxide anion production by polynuclear leucocytes from patients with rheumatoid arthritis. J Rheumatol 1995;22:633-638

14. Cutolo M, Sulli A, Pizzorni C and Seriolo B. Antiinflammatory mechanisms of methotrexate in rheumatoid arthritis. Ann Rheum Dis 2001;60:729-735.

15. Gutteridge JM. Antioxidant properties of the proteins ceruloplasmin, albumin and transferrin. A study of their activity in serum and synovial fluid from patients with rheumatoid arthritis. Biochem Biophys acta 1986;869:119-127.

16. Deneke SM and Fanburg BL. Regulation of cellular glutathione. Am J Physiol 1989;257:L163-173.

17. Fridovich I. Superoxide dismutases.Annu Rev Biochem 1975;44:147-159. Wong GH and Goeddel DV. Induction of manganous superoxide dismutase by tumour necrosis factor: possible protective mechanism. Science 1988;242:941-944.

18. Nivsarkar M. Improvement in circulating superoxide dismutase levels: role of non-steroidal antiinflammatory drugs rheumatoid arthritis. Biochem Biophys Res commun 2000;270:714-716. 